\title{
ON MODEling CONTROLleR-SWitCH INTERACTION IN OPENFLOW BASED SDNS
}

\author{
Ameen Chilwan ${ }^{1,}$ Kashif Mahmood $^{2}$, Olav N. Østerb $\varnothing^{2}$ and Michael Jarschel ${ }^{3}$ \\ ${ }^{1}$ Department of Telematics, NTNU, Norway \\ ${ }^{2}$ Telenor Research, Norway \\ ${ }^{3}$ Nokia, Germany
}

\begin{abstract}
With an increase in number of software defined network (SDN) deployments, and OpenFlow consolidating as the protocol of choice for controller-switch interactions, a need to analytically model the system for performance analysis is increasing. An attempt has previously been made in [1] to model the system considering both a controller and a switch as an $M / M / 1$ queue. The method, although useful, lacks accuracy for higher probabilities of new flows entering the network. The approach is also deficient of details on how it can be extended to more than one node in the data plane

These two short-comings are addressed in this paper where the controller and switch are modeled collectively as Jackson's network, with essential tuning to suit OpenFlow-based SDN. The consequent analysis shows the resilience of the model even for higher number of new flow entries. An example is also used to illustrate the case of multiple nodes in the data plane.
\end{abstract}

\section{KEYWORDS}

OpenFlow, Performance analysis, Queuing theory, Software defined networks.

\section{INTRODUCTION}

Software defined networking (SDN) is a networking concept which involves separating the control plane from the data plane. SDN promises network deployment and service upgrade on software time scales which has huge benefits especially in the carrier network domain because in the future the network operators will not compete on the basis of network coverage alone but on the basis of features and services.

The initial impact of SDN was seen in the datacenters. As early as January 2012, Google had their full scaled datacenter WAN running as OpenFlow based SDN [2]. SDN is now all set to roar in the wireless domain too. All this has been possible due to the basic architectural principle of SDN which is the separation of the control plane from the data plane. The architecture involves SDN controller(s) residing in the control plane while the forwarding element(s) make the data plane. In order to handle the communication between the control plane and the data plane elements, OpenFlow is the only open, standard protocol [3].

OpenFlow started as a test protocol in Stanford but is now managed and maintained by Open Networking Foundation (ONF) [3]. It started with OpenFlow version 1.0.0 and at the writing of the paper, version 1.4 has been specified [3]. The working principle is the same but each version involves some additional features. For example the version 1.1.0 has support for group tables

DOI : $10.5121 /$ ijcnc.2014.6611 
which was not there in version 1.0.0. The work in this paper is based on OpenFlow version 1.0.0 and we believe that it can be easily extended to the new versions.

Under an OpenFlow network, the controller-to-switch communication takes place as follows: where we use the term switch and node interchangeably to represent the forwarding element in the data plane in an SDN network.

When a flow with no specified forwarding instructions comes into a network the following actions are taken:

i. A packet (or part of the packet) of the flow is sent by the switch to the controller, assuming that the switch is not configured to drop unknown packets.

ii. The controller computes the forwarding path and updates the required nodes in the data path by sending entries to be added to the flow tables.

iii. All subsequent packets of the flow are forwarded based on pre-calculated forwarding decisions and do not need any control plane action.

It is important to model the controller-to-switch communication for the performance analysis of OpenFlow (OF)-based SDN networks. The modeling of OpenFlow networks will help us to answer questions such as how much data we can pump into the network, what is the packet sojourn time, when and what (switch or the controller) is the bottleneck in a network.

Most of the work on performance analysis of SDN networks is based on simulations or experimentations. Albeit their benefits, analytical modeling is a time efficient alternative because setting up an SDN experiment or performing a simulation can take hours. The analytical model for an OF-based SDN should be able to capture the OpenFlow working principle and at the same time shall be flexible to handle any amount of query traffic going to the controller. Further, the model shall be readily extendable to more than one node in the data plane.

The analytical modeling of OpenFlow-based networks has only been attempted in a handful of papers before. For example feedback oriented queuing theory has been used in [1] to capture the control plane and data plane interaction where the Markovian servers are assumed for both the controller and the switch. However the model becomes less accurate as the probability of traffic going to the controller increases. Secondly it is not clear how the model can be extended to more than one switch in the data plane.

In [4] a network calculus based approach is used to quantify the packet processing capability of the switch in the data plane. However the feedback between the nodes in the data plane and the controller is not considered. This shortcoming of feedback modeling is addressed in [5]. However the model is depicted only for a single node in the data plane and the time stopping method employed therein has limited real time application. Secondly the framework used in [4] and [5] is based on deterministic network calculus which does not provide any meaningful bounds [6]. To the best of our knowledge apart from these handful analytical works, almost all the other efforts of evaluating performance of OpenFlow-based networks are carried out by simulations or measurements, for example [7], [8], [9]. Moreover, Cbench tool to benchmark the controller performance is also introduced in [10] and is proved to be instrumental in benchmarking.

It is therefore of paramount importance to have an analytical model which can capture the feedback interaction between the controller and the switch, is able to model any amount of traffic going from switch to controller (and vice versa), and can be easily extended to more than one switch in the data plane. The model proposed in this paper is an attempt in that direction. We model the OpenFlow network as a Jackson network but with a modification to accurately represent the traffic flow from the switch to the controller in an actual OF-based SDN network. It 
needs to be emphasized that this model was first presented by us in [11]. Here in this work we elaborate the model with more performance measures.

It is highlighted later in the paper (Fig. 2) that this modification to the native Jackson network is necessary to capture the OpenFlow working principle. The model is in turn used for performance analysis of OF-based SDN networks to calculate the mean packet sojourn time, to find out how much data we can pump into the network and when and what (switch or the controller) is the bottleneck in a network. The main contributions of this work are:

- A model is proposed to capture the feedback interaction between the switch and the controller mimicking an actual OpenFlow based SDN network

- The model is accurate even for the case when large amount of new flows are arriving at the switch.

- The model can be easily extended to more than one switch in the data plane.

- We show mathematically that the packet sojourn time calculated by our proposed model based on Jackson assumption is the same as the one explicitly calculated for OF-based SDN network in [1].

The rest of the paper is organized as follows; we first present the system model along with the limitations and the necessary preliminaries in Section 2. The performance measures are outlined in Section 3, while the numerical results are presented in Section 4. An insight as to how the proposed model can be used for multi-node case is highlighted in Section 5, while the conclusions along with the future research directions are presented in Section 6.

\section{MODEL DESCRIPTION}

We assume that the overall traffic process at the switch and at the controller follows Poison process similar to [1] given that the two processes are on a different time scale. Further we assume Markovian servers for the switch and the controller wherein we incorporate the transmission time of the packets from the switch to the controller in the service time of the controller. As for the buffer size we assume infinite buffer for the switch while for the controller we initially do the analysis based on infinite buffer and later in the paper we extend it to the finite buffer case.

We use Jackson network model to represent the OF-based SDN network. To this end a recap of the Jackson model for open queuing networks [12], in which the nodes behaves locally as single $\mathrm{M} / \mathrm{M} / 1$ queues, is outlined. Albeit trivial, this is done in order to highlight that Jackson model cannot be used as such for modeling OF-based SDNs.

Let us consider a Jackson network consisting of two nodes 1 and $c$ connected in a feedback path as shown in Fig. 1(a). The service rates of nodes $l$ and $c$ are exponentially distributed with average values of ${ }_{l}$ and ${ }_{c}$, respectively. The external traffic arrival to the node $l$ is denoted as $\lambda_{l}$ packets per unit time.

Let $\Gamma_{1}$ be the net input to node 1 out of which $\Gamma_{c}=q_{1}^{j a c k} \Gamma_{1}$ goes to the node $c$ where $q_{1}^{\text {jack }}$ is the probability that the packet goes to the node $\mathrm{c}$.

Assuming that no packet is lost at the controller (infinite buffer at the controller) the balance equation for the system can be written as

$$
\Gamma_{1}=\lambda_{1}+q_{1}^{j a c k} \Gamma_{1}
$$




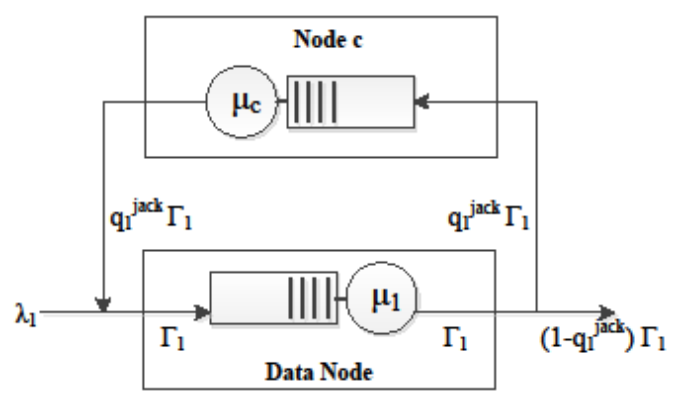

Figure 1(a): Jackson Model

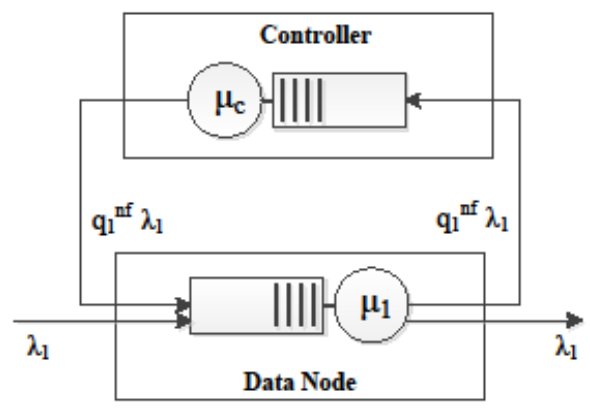

Figure 1(b): Simple OpenFlow Model [1]

It is the term $q_{1}^{\text {jack }}$ which needs modification in order to model OF-based SDN in Fig. 1(b) as a Jackson network in Fig. 1(a). This is because an OF-based SDN, as shown in Fig. 1(b), has the following two salient features:

i. A packet coming to any node in the data plane will at most visit the controller once.

ii. Only a fraction of the external traffic $\lambda_{l}$ and not a fraction of the net input traffic $\Gamma_{l}$ will go the controller. (Two lines directly out of the data node in Fig. 1(b) as opposed to one line in Fig. 1(a) are used to represent this phenomenon).

In an OpenFlow network, let $q_{1}^{n f}$ be the probability that the packet goes to the controller in case there is no flow entry in flow-table of the node, then in order to use the Jackson network to represent the OF-based SDN we have to adjust $q_{1}{ }^{j a c k}$ by demanding that the input rates to the nodes in both the models are the same. Hence

and

$$
\Gamma_{1}=\lambda_{1}+q_{1}^{n f} \lambda_{1}
$$

$$
q_{1}^{\text {jack }} \Gamma_{1}=q_{1}^{n f} \lambda_{1}
$$

As a result $q_{1}^{\text {jack }}$ can be solved as

$$
q_{1}^{j a c k}=\frac{q_{1}^{n f}}{1+q_{1}^{n f}}
$$

Fig. 2 highlights the need of having a modified Jackson model to represent OF-based SDN networks where we represent mean time spent by a packet in the network (node + controller) as a function of load on the controller.

The curve simulation is obtained from simulating the OpenFlow behavior taking into account the aforementioned two salient features. Further, in the simulation, we assume Poisson arrivals at the input and exponentially distributed service times for the nodes.

The curve denoted by Jackson Model is obtained by using $q_{I}^{\text {jack }}$ as such without modification i.e. $q_{I}^{j a c k}=q_{I}{ }^{\text {jf }}$ while the curve Modified Jackson Model is based on $q_{I}{ }^{\text {jack }}$ in (4).

It can be seen that as the percentage of traffic going to the controller dictated by $q_{1}{ }^{n f}$ increases, the modification to the probability $q_{1}^{\text {jack }}$ in (4) becomes all the more important. 


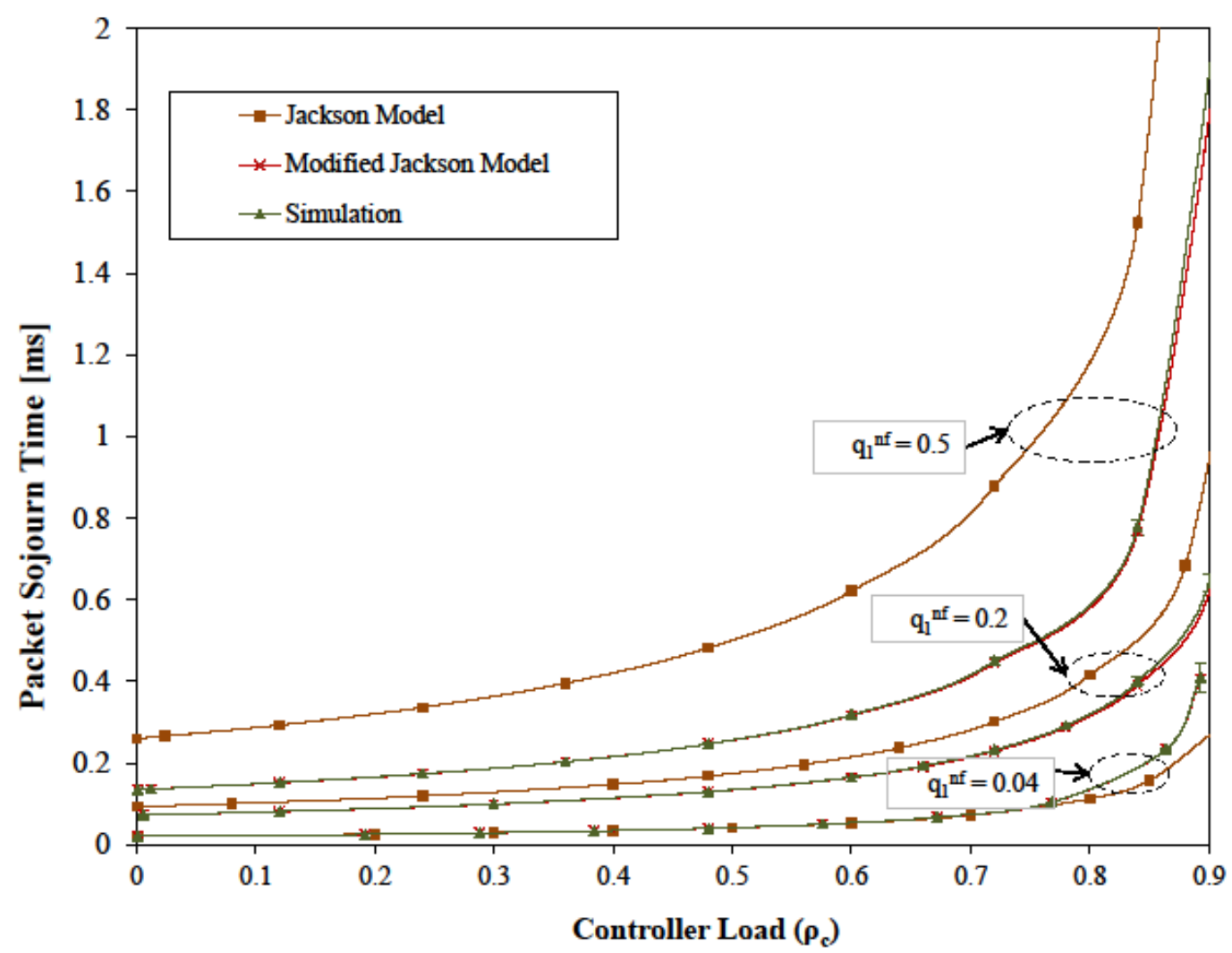

Figure 2: Jackson model without modification overestimates the mean packet sojourn time

\subsection{Limitations}

The work in this paper makes the following assumptions:

- The overall traffic arrival process at the switch and the controller is Poison. Further exponentially distributed service times are used for the switch and the controller. This allows us to use the Jackson network results based on $\mathrm{M} / \mathrm{M} / 1$ queues.

- Secondly we assume a single queue at the switch instead of a separate queue per line card.

- TCP traffic is used for which only the first packet of the unknown flow is sent to the controller.

- Infinite buffer is assumed at the switch as typically it is quite large.

It needs to be emphasized that the main goal of this work is to develop an analytical model for OF-based SDN networks. The assumptions will be relaxed in the subsequent work.

\section{Performance Measures}

One of the advantages of our proposed model is that we can leverage the well-established results for performance analysis of Jackson networks for analysing OpenFlow-based SDNs. In this section, we will use the proposed model to find two elementary performance measures, the average packet sojourn time, and the distribution of time spent by a packet in the network. 


\subsection{Average Packet Sojourn Time}

The average packet sojourn time, $E\left[W^{\text {jack }}\right]$, is defined as the time spent by a packet in the network from the moment it enters the network at its source node, until it leaves through the destination. $E\left[W^{\text {jack }}\right]$ for the network in Fig. 1 is given as [12]

$$
E\left[W^{j a c k}\right]=\frac{1}{\lambda_{1}} / \frac{\rho_{1}}{1-\rho_{1}}+\frac{\rho_{c}}{1-\rho_{c}} /
$$

where $\rho_{1}=\Gamma_{l} /_{l}$ and $\rho_{c}=\Gamma_{c}{ }_{c}$ denote the load on the node 1 and the controller $c$, respectively. Further, in order to have a stable system, it is assumed that all the loads are less than unity that is $\rho_{l}<1$ and $\rho_{c}<1$.

Alternatively, we can use the delay formula derived explicitly for the OpenFlow model, depicted in Fig. 1(b), as highlighted in [1].

To this end it needs to be highlighted that a packet arriving at the switch in the data plane of the OpenFlow network is confronted with two conditions. If there is already a flow entry installed in the switch then the packet is forwarded as such after spending time $T_{l}$ otherwise it goes to the controller, spends time $T_{c}$ then returns back to the same switch for packet matching where it spends time $T_{1}^{(2)}$ and is forwarded on the output interface.

So the absolute value of packet sojourn time $W^{\text {of }}$ in an OF-based SDN network where node 1 interacts with the SDN controller $c$ as shown in Fig. 1(b) is given as

$$
W^{o f}= \begin{cases}T_{1} & \text { with probability } 1-q_{1}^{n f} \\ T_{1}+T_{c}+T_{1}^{(2)} & \text { with probability } q_{1}^{n f}\end{cases}
$$

where $T_{1}$ and $T_{c}$ are sojourn times in node 1 and node $c$ respectively, while $T_{1}^{(2)}$ is the sojourn time when a packet enters node 1 the second time after visiting the controller.

Eventually, the mean of $W^{\text {of }}$ is given as

$$
\begin{aligned}
E\left[W^{o f}\right] & =\left(1-q_{I}^{n f}\right) E\left[T_{1}\right]+q_{1}^{n f}\left(E\left[T_{1}\right]+E\left[T_{c}\right]+E\left|T_{l}^{(2)}\right|\right) \\
& =\left(1+q_{1}^{n f}\right) \frac{1}{{ }_{{ }_{1}}-\Gamma_{1}}+q_{1}^{n f} \frac{1}{c^{-} \Gamma_{c}}
\end{aligned}
$$

We draft a short proof in Lemma 1 to show that the mean packet sojourn time calculated by the two methods is indeed the same.

Lemma 1: For the single node case the packet sojourn time calculated in (5) using the standard Jackson assumption is the same as explicitly calculated using (7).

Proof: By rearranging (7) in terms of traffic loads we have:

$$
E\left[W^{o f}\right]=\frac{1+q_{1}^{n f}}{\Gamma_{1}} / \frac{\rho_{1}}{1-\rho_{1}} /+\frac{q_{1}^{n f}}{\Gamma_{c}} / \frac{\rho_{c}}{1-\rho_{c}} /
$$


Using $\Gamma_{1}=\left(1+q_{1}^{n f}\right) \lambda_{1}$ from (2) and $\Gamma_{c}=q_{1}^{n f} \lambda_{1}$ from (3) we obtain $E\left[W^{j a c k}\right]$, in (5) which proves the Lemma.

\subsection{Distribution of Time Spent by the Packet}

In this section we take a step forward by presenting the probability density function (PDF) and the cumulative density function (CDF) of the time spent by a packet in the node.

Lemma 2: The $\operatorname{PDF} w_{l}^{c}(t)$ and the $\operatorname{CDF} \widetilde{W}_{l}^{c}(t)$ of the time spent by a packet in the node 1 are given respectively as

$$
\begin{aligned}
w_{l}^{c}(t) & =b_{1}^{(1)} a_{1} e^{-a_{1} t}+b_{1}^{(2)} a_{1}\left(a_{1} t\right) e^{-a_{1} t}+d_{1} a_{c} e^{-a_{c} t} \\
\widetilde{W}_{1}^{c}(t) & =P\left(W^{o f}>t\right)=\left(b_{1}^{(1)}+b_{1}^{(2)}\right) e^{-a_{1} t}+b_{1}^{(2)}\left(a_{1} t\right) e^{-a_{1} t}+d_{1} e^{-a_{c} t}
\end{aligned}
$$

where

$a_{1}={ }_{1}-\Gamma_{1}, a_{c}={ }_{c}-\Gamma_{c}$

while

$b_{1}^{(1)}=1-q_{1}^{n f}-q_{1}^{n f} \frac{a_{1} a_{c}}{\left(a_{c}-a_{1}\right)^{2}}, \quad b_{1}^{(2)}=q_{1}^{n f} \frac{a_{c}}{a_{c^{-}} a_{1}}, \quad d_{1}=q_{1}^{n f} \frac{a_{1}^{2}}{\left(a_{c}-a_{1}\right)^{2}}$

Proof: If we assume that the sojourn times $T_{1}$ and $T_{1}^{(2)}$ are independent then the Laplace transform $W_{l}^{c}(s)=E\left[e^{-s W^{o f}}\right]$ may be written as

$$
W_{1}^{c}(s)=\left(1-q_{1}^{n f}\right) \frac{a_{1}}{a_{1}+s}+q_{1}^{n f}\left(\frac{a_{1}}{a_{1}+s}\right)^{2}\left(\frac{a_{c}}{a_{c}+s}\right)
$$

which can further be written as

$$
W_{l}^{c}(s)=b_{l}^{(1)} \frac{a_{1}}{a_{1}+s}+b_{l}^{(2)}\left(\frac{a_{1}}{a_{1}+s}\right)^{2}+d_{l}\left(\frac{a_{c}}{a_{c}+s}\right)
$$

Inverting the Laplace transform proves the Lemma.

\subsection{Finite buffer at the controller: The $M / M / 1-S$ case}

We relax the assumption of having infinite buffer at the controller. Instead we assume that the buffer space at the controller is limited to at most $S$ places and the rest remains the same. Let $p_{b}$ be the probability that the packet is blocked at the controller. For this model (M/M/1-S) the distribution of the number of packets $j_{c}$ is given as

$$
P\left(j_{c}\right)=\frac{1-\rho_{c}}{1-\rho_{c}^{S+1}} \rho_{c}^{j_{c}}
$$

Hence $p_{b}$ which is the probability that the buffer is full can be written by replacing $j_{c}$ with $S$ in (13). Further the mean numbers of packets $E\left[N_{c}\right]$ in the controller is found as [12] 
International Journal of Computer Networks \& Communications (IJCNC) Vol.6, No.6, November 2014

$$
E\left[N_{c}\right]=\frac{\rho_{c}}{1-\rho_{c}^{S+1}}\left(\frac{1-\rho_{c}^{S+1}}{1-\rho_{c}}-S \rho_{c}^{S}\right)
$$

where the load on the controller $\rho_{c}$ is given as $\rho_{c}=\Gamma_{c}{ }_{c}$ with $\Gamma_{c}=q_{1}^{\text {jack }} \Gamma_{l}$ being the traffic going to the controller.

Finally by applying Little's formula [12], the sojourn time of a packet in controller is calculated as

$$
E\left[T_{c}\right]=\frac{E\left[N_{c}\right]}{\Gamma_{c}\left(1-p_{b}\right)}
$$

Applying the rate equivalence detailed earlier and incorporating $p_{b}$ in the balance equation for $\Gamma_{l}$ we get $q_{1}^{j a c k}$ for the finite buffer case as

$$
q_{1}^{j a c k}=\frac{q_{1}^{n f}}{1+q_{1}^{n f}\left(1-p_{b}\right)}
$$

\section{NUMERICAL RESUltS}

In order to verify our proposed model we developed a discrete event simulation model to mimic the queuing behavior in an OF-based SDN. We assume that at the arrival to the node 1 , packets are queued in the data node before being processed. The processing time of data node $1 /{ }_{1}$ is considered to be exponentially distributed with a mean value of $9.8 \mathrm{~s}$. The value of 9.8 is the average processing time taken by Pronto 3290 switch for forwarding packets of size 1500 bytes [1]. We here assume that TCP uses maximum transmission unit (MTU) of 1500 bytes.

At the controller, the number of responses per second are taken to be 4175 as reported in [1] by using the Cbench tool [10]. Hence this is parameterized as $1 /{ }_{c}=240 \mathrm{~s}$ in the model.

To enhance confidence in the simulation result, five replications for each value were run and the normally distributed $95 \%$ confidence interval is incorporated in the plots.

We first highlight in Fig. 3 that our proposed model provides a fix to the results reported by [1]. To this end we first plot the Simulation curve from [1] as a reference. On top of it we plot the analytical curves; Analytical [1] and Modified Jackson Model, obtained using the model in [1] and our proposed Jackson model, respectively. 
International Journal of Computer Networks \& Communications (IJCNC) Vol.6, No.6, November 2014

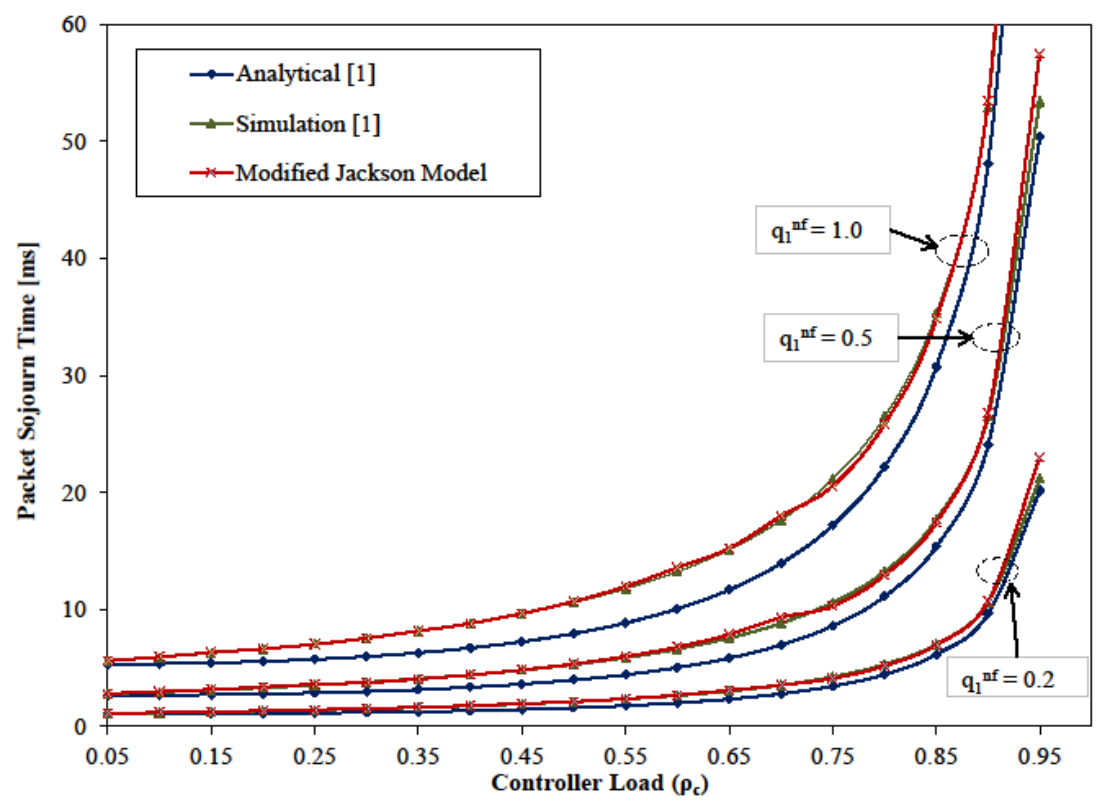

Figure 3: Comparing Modified Jackson Model to Results from [1]

It can be seen that the model proposed by [1] performs very well for small loads $\left(q_{1}{ }^{n f}=0.2\right)$. However in the cases when there is a large amount of query traffic coming to the controller due to unknown flows the model in [1] falls short. In such cases the proposed modification to the Jackson model is quite accurate as seen for the extreme case of $q_{1}{ }^{n f}=1.0$.

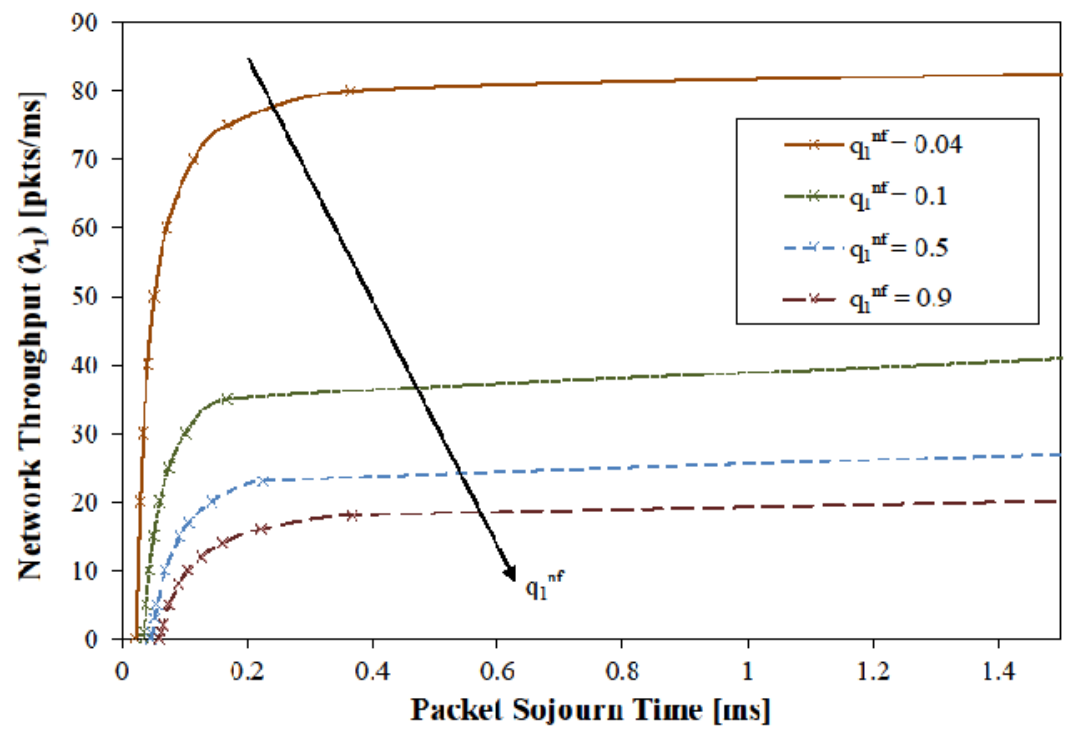

Figure 4: Dimensioning Network Throughput

In Fig. 4, the effect of $q_{1}^{n f}$ on network throughput is studied where the network throughput is defined as the amount of traffic $\lambda_{1}$ which can be injected into the OF-based SDN for a given delay guarantee. In this case the delay guarantee is the average packet sojourn time. This plot also highlights how the proposed model can be used to dimension the network if packet sojourn time is considered as the design parameter. A striking feature of this plot is that the network 
throughput saturates after reaching a certain value of packet sojourn time. Subsequently, it can be inferred that even if the network is over-loaded after crossing a certain traffic threshold, the result will be just increased packet sojourn time without further enhancing the network throughput. Similarly, it is also observed that the critical value for packet sojourn time remains almost the same for all the values of $q_{1}^{n f}$ but the resulting network throughput for each of them is quite different.

In Fig. 5 a fundamental performance plot is shown in which packet sojourn time is plotted against the controller load $\rho_{\mathrm{c}}$ for differing values of the controller service time ${ }_{\mathrm{c}}$ with $\mathrm{q}_{1}{ }^{\text {nf }}$ constant at 0.5 . Although the plot is mainly for evaluating performance, it can also play a role in designing a network with controller of known average service time and giving guarantees on packet sojourn time by keeping the controller load at a certain level.

In Fig. 6, the plot shows the probability that packet sojourn time $\leq 0.5 \mathrm{~ms}$ for varying values of controller load $\rho_{c}$ and for different $q_{1}{ }^{n f}$ values. The plot can be used to determine the maximum load that the controller should reach before its performance is compromised. The plot in Fig. 6 is pilot and similar plots for different values of packet sojourn time can be obtained depending upon the requirements.

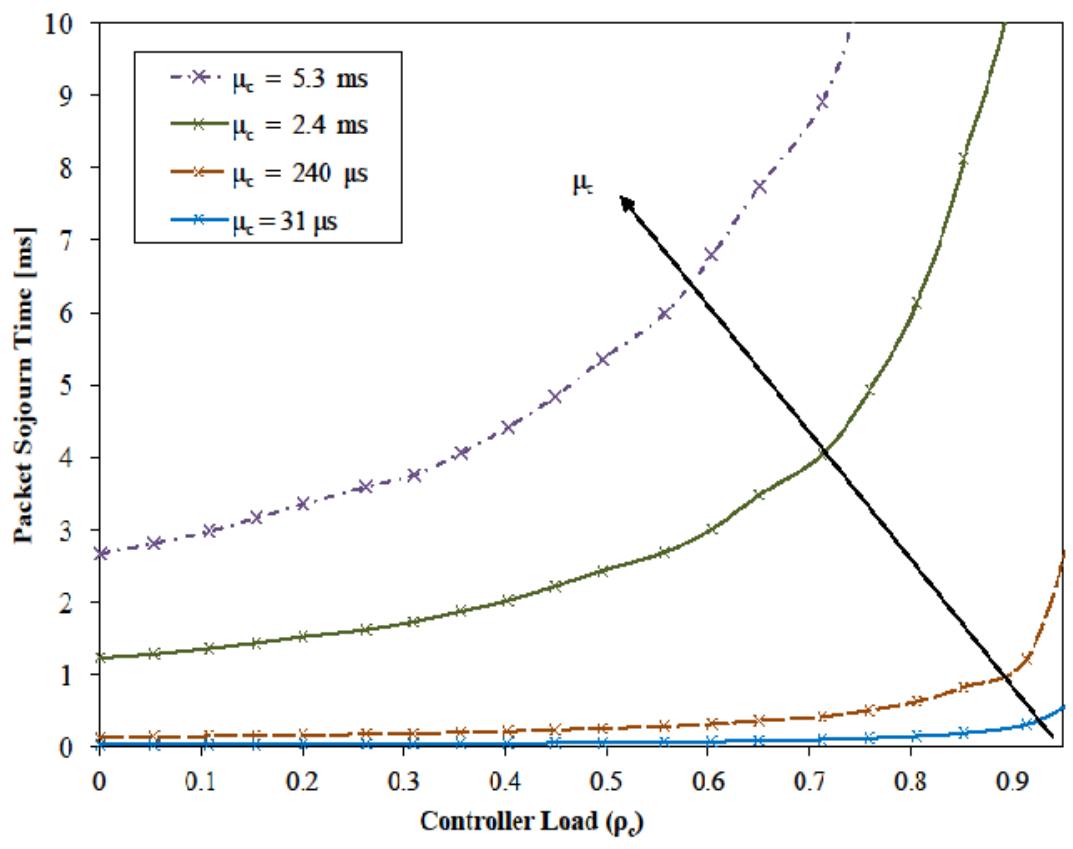

Figure 5: Effect of the controller service rate ${ }_{c}$ 
International Journal of Computer Networks \& Communications (IJCNC) Vol.6, No.6, November 2014

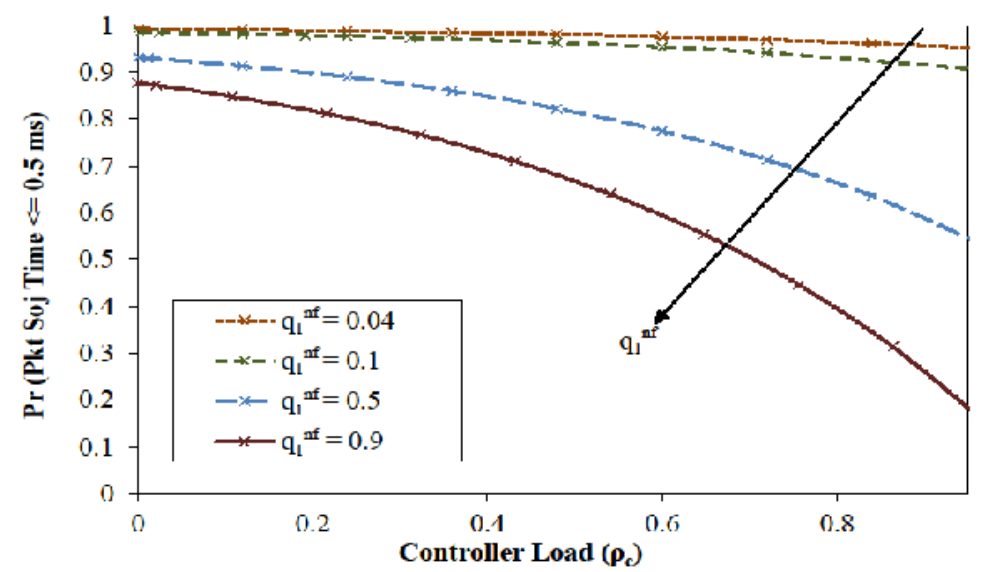

Figure 6: CCDF of packet sojourn time as a function of load on controller

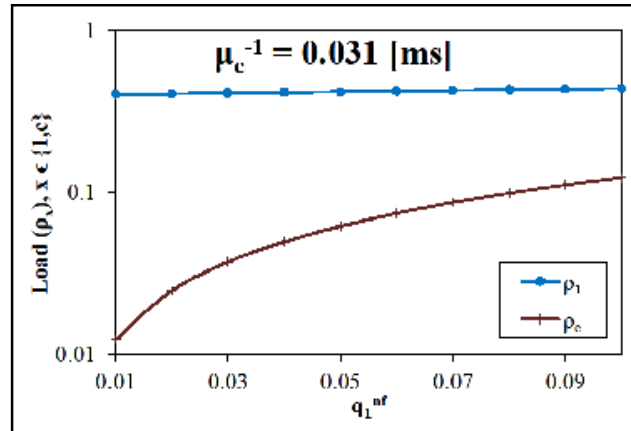

(a) Node is Bottleneck

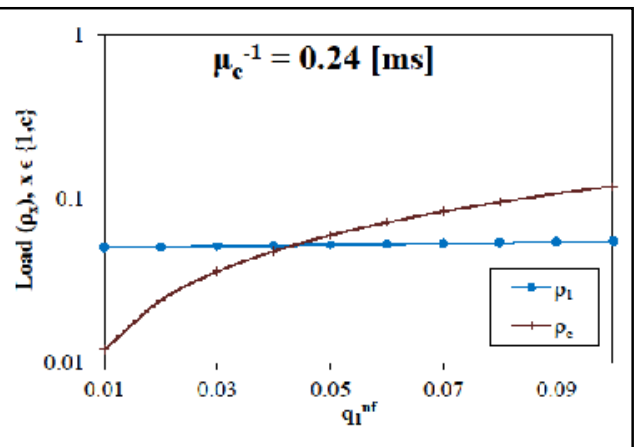

(b) Node/Controller is Bottleneck

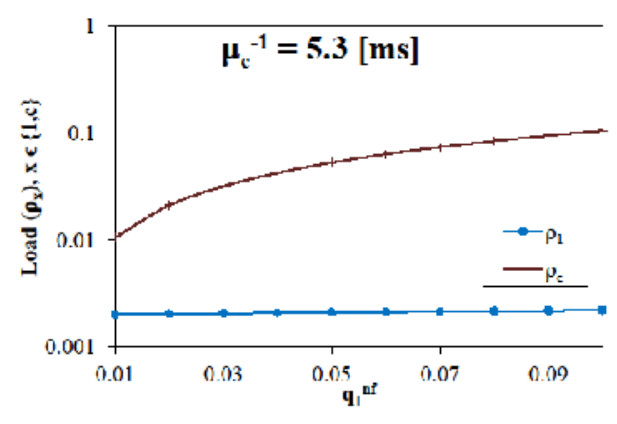

(c) Controller is Bottleneck

Figure 7: Bottleneck: the Controller or the Data Node?

Finally in Fig. 7 we plot the load on both the switch and the controller against $q_{1}^{n f}$ for different values of mean controller service rate ${ }_{c}$. By doing so, the sensitivity of the load on the switch and the controller for varying values of $q_{1}^{n f}$ are inferred. Furthermore this plot can also be helpful in determining the bottleneck node for different network settings. Hence, for a specific value of controller service time, it can be determined by using Fig. 7 that which node is actually service bottleneck and should be selected intelligently. For example for the average controller service 
times ( ${ }_{c}$ ) of $31 \mathrm{~s}$, the switch is the bottle neck while for $5.3 \mathrm{~ms}$ it is the controller. However for the case when the mean service time of the controller is $240 \mathrm{~s}$, the switch is the bottleneck for approximately $q_{1}{ }^{n f} \leq 0.04$ while it is the controller when $q_{1}{ }^{n f}>0.04$.

It needs to be emphasized that blocking probability $p_{b}$ was zero for the setup which we had for the simulation and infinitesimal small for the analytical model.

\section{The Multi-Node CASE}

In a real life SDN deployment, an SDN controller is responsible for more than one node in the data plane. In this section we highlight how the proposed model can be used to model this scenario. To this end we take a toy example in which we only have two nodes in the data plane as shown in Fig. 8. We define $q_{2}^{j a c k}$ and $q_{2}{ }^{n f}$ for node 2 similar to $q_{1}^{j a c k}$ and $q_{1}{ }^{n f}$ defined earlier for node 1 .
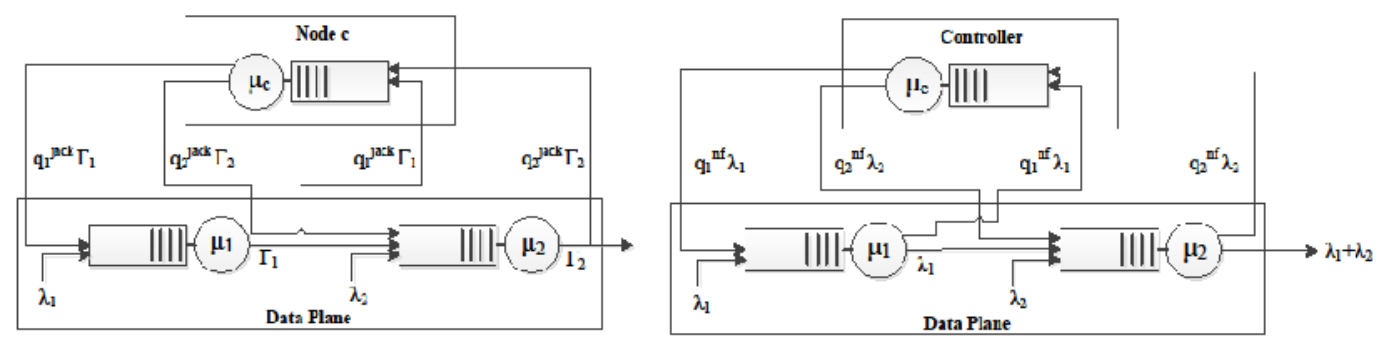

Figure 8: Modeling more than one node in the data plane

In order to leverage the Jackson model in Fig. 8(a) for modeling the OF-based SDNs in Fig. 8(b), the probabilities $q_{2}{ }^{\text {jack }}$ and $q_{1}^{\text {jack }}$ need to be adjusted. This is accomplished by forcing the rates at all the nodes in both the models to be the same as

For node $1: \quad \lambda_{1}\left(1+q_{1}^{n f}\right)=\Gamma_{1}, \quad q_{1}^{n f} \lambda_{1}=q_{1}^{j a c k} \Gamma_{1}$

(b) Multi-node OpenFlow Model

For node 2: $\quad \lambda_{1}+\lambda_{2}\left(1+q_{2}^{n f}\right)=\Gamma_{2}, \quad q_{2}^{n f} \lambda_{2}=q_{2}^{\text {jack }} \Gamma_{2}$

Solving (17) we get $\mathrm{q}_{1}{ }^{\text {jack }}$ same as (4) while by solving (18) we have $\mathrm{q}_{1}{ }^{\text {jack }}$ as

$$
q_{2}^{j a c k}=\frac{q_{2}^{n f} \lambda_{2}}{\lambda_{1}+\lambda_{2}\left(1+q_{2}^{n f}\right)}
$$

We can then use the modified $q_{1}^{j a c k}$ and $q_{2}^{\text {jack }}$ to derive the appropriate performance metrics such as packet sojourn time using existing queuing theory results [12] similar to the single node case.

\section{CONCLUSION}

In this work we have proposed an analytical model for an OpenFlow enabled SDN based on Jackson network. We have shown that the model is accurate even for the case when the probability of new flows is quite large. The applicability of the model is determined by establishing two performance measures, the average packet sojourn time and the distribution of time spent by a packet in the network, by using the proposed model. Secondly we showed by a 
toy example that the model can be readily extended to more than one switch in the data plane. Conclusively it is noted, and can be safely stated, that the model proposed in this paper caters for realistic OpenFlow-based SDNs and this argument has readily been validated in this paper.

Furthermore, the effects of key parameters in an SDN network are studied which include the time required by the controller to process a request, amount of traffic going to the controller, average time spent by a packet in a network and the network throughput.

There is more than one direction that the work presented in this paper can be taken forth. First of all, the work presented and validated for a single node can be extended to larger and more realistic topological scenarios, such as fat-tree topology. Secondly, the model in this work is based on Markovian arrival and service processes which can be generalized and more realistic distributions or traces can be used in modeling. This can be supplemented with simulations for validation and verification of the model. Also, a test-bed study for verifying our model can be performed which will enhance the confidence in the proposed model.

\section{REFERENCES}

[1] Jarschel, Michael \& Oechsner, Simon \& Schlosser, Daniel \& Pries, Rastin \& Goll, Sebastian \& Tran-Gia, Phuoc (2011) "Modeling and Performance Evaluation of an OpenFlow Architecture", Proceedings of the 23rd International Teletraffic Congress (ITC '11), pp1-7.

[2] Hoelzle, Urs (2012) “Opening Address: 2012 Open Network Summit", [Online] Available: http://www.opennetsummit.org/archives/apr12/hoelzle-tue-openflow.pdf, Date Retrieved: 08/08/2014.

[3] ONF, Open Networking Foundation, [Online] Available: https://www.opennetworking.org.

[4] Bozakov, Zdravko \& Rizk, Amr (2013) "Taming SDN Controllers in Heterogeneous Hardware Environments", 2nd IEEE European Workshop on Software-Defined Networks (EWSDN).

[5] Azodolmolky, Siamak \& Nejabati, Reza \& Pazouki, Maryam \& Simeonidou, Dimitra (2013) "An Analytical Model for Software Defined Networking: A Network Calculus-based Approach”, IEEE Globecom.

[6] Ciucu, Florin \& Schmitt, Jens (2012) "Perspectives on Network Calculus: No Free Lunch, but Still Good Value", SIGCOMM Computer Communication Reviews, vol. 42, no. 4, pp 311-322.

[7] Naous, Jad \& Erickson, David \& Covington, G. Adam \& Appenzeller, Guido \& McKeown, Nick (2008) "Implementing an OpenFlow Switch on the NetFPGA Platform", Proceedings of the 4th ACM/IEEE Symposium on Architectures for Networking and Communications Systems (ANCS 'O8), pp 1-9.

[8] Bianco, Andrea \& Birke, Robert \& Giraudo, Luca \& Palacin, Manuel (2010) "OpenFlow Switching: Data Plane Performance", IEEE International Conference on Communications (ICC) 2010, pp 1-5.

[9] Khan, Asif \& Dave, Nirav (2013) "Enabling Hardware Exploration in Software-Defined Networking: A Flexible, Portable OpenFlow Switch", IEEE 21st Annual International Symposium on Field-Programmable Custom Computing Machines (FCCM), 2013.

[10] Sherwood, Rob "Cbench (Controller benchmarker)", [Online] Available: http://archive.openflow.org/wk/index.php/Oflops, Date Retrieved: 13/03/2014.

[11] Mahmood, Kashif \& Chilwan, Ameen \& Østerbø, Olav N. \& Jarschel, Michael (2014) "On the Modeling of OpenFlow-based SDNs: The Single Node Case", Proceedings of the Sixth International Conference on Networks and Communications (NeCOM 2014), CS \& IT-CSCP 2014, vol. 4, pp 207-214, [Online] Available: http://airccj.org/CSCP/vol4/csit42920.pdf.

[12] Jackson, James R. (1957) "Networks of Waiting Lines”, Operations Research, vol. 5, no. 4, pp 518-521. 


\section{AUTHORS}

Ameen Chilwan is currently pursuing his $\mathrm{PhD}$ in Telematics at the Norwegian University of Science and Technology (NTNU) from where he did his MSc too in 2011. Previously, he received BSc in Telecommunications Engineering from the University of Dar es Salaam (UDSM). His current work focuses on providing network infrastructure with guaranteed delays for distributed media plays. His research interests also include modelling and performance analysis of communication systems, dependability modelling, buffer management, real-time internet, cloud computing and SDNs.

Kashif Mahmood is a Research Scientist at Telenor Research in Norway. He received his MS in Electronics from Ghulam Ishaq Khan Institute (GIKI), and $\mathrm{PhD}$ degree in Telematics from Norwegian university of science and technology (NTNU), Norway. Kashif is a recipient of the "Youth Award in Science and Technology" by the government of Pakistan. His work on radio wave propagation modelling using semi deterministic approach was awarded the best paper presentation award in ISAP 2007 while the work on

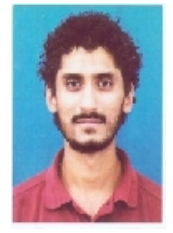
cross layer modelling of CDMA networks won the best student paper award in ICON 2011.His research interests include cross layer modelling of communication networks, NFV and SDN, scheduling algorithms, traffic modelling and M2M.

Olav N. Østerbø received his MSc in Applied Mathematics from the University of Bergen in 1980 and his PhD from the Norwegian University of Science and Technology in 2004. He joined Telenor in 1980 and has more than 30 years of experience in telecom research. Activities in recent years have been related to QoS and performance analysis. Current research topics include traffic modelling, analysis of interference in radio networks, scheduling, traffic differentiation and M2M.

Michael Jarchel is working as a research engineer in the area of Software Defined Networking at Nokia in Munich, Germany. He finished his Ph.D. thesis, titled "An Assessment of Applications and Performance Analysis of Software Defined Networking", at the University of Würzburg in 2014. His main research interests are in the applicability of SDN and NFV concepts to next generation mobile networks.
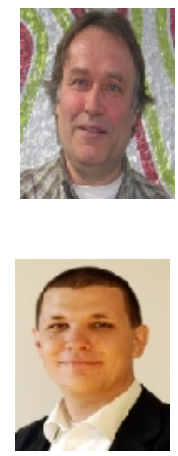